\title{
Transverse-to-longitudinal emittance exchange to improve performance of high-gain free-electron lasers
}

\author{
P. Emma and Z. Huang \\ Stanford Linear Accelerator Center, Stanford, California 94309, USA \\ K.-J. Kim \\ Argonne National Laboratory, Argonne, Illinois 60439, USA \\ P. Piot \\ Northern Illinois University, DeKalb, Illinois 60115, USA \\ and Fermi National Accelerator Laboratory, Batavia, Illinois 60510, USA
}

(Received 1 August 2006; published 25 October 2006)

\begin{abstract}
The ability to generate small transverse emittance is perhaps the main limiting factor for the performance of high-gain x-ray free-electron lasers (FELs). Noting that beams from an rf photocathode gun can have energy spread much smaller than required for efficient FEL interaction, we present a method to produce normalized transverse emittance at or below about $0.1 \mu \mathrm{m}$, which will lead to a significantly shorter length undulator as well as a lower electron beam energy for an x-ray FEL project. The beam manipulation consists of producing an unequal partition of the initially equal emittances into two dissimilar emittances by a flat-beam technique and exchanging the larger transverse emittance with a smaller longitudinal emittance. We study various issues involved in the manipulation. In particular, a new emittance exchange optics we found enables an exact emittance exchange necessary for this scheme.
\end{abstract}

DOI: 10.1103/PhysRevSTAB.9.100702

PACS numbers: 29.27.-a, 29.27.Eg, 41.60.Cr

\section{INTRODUCTION}

Accelerated beams often need to be manipulated in phase space to optimize their efficiency for application. It has been noted [1] that the phase-space distribution of an electron beam from a laser-driven rf photocathode is not ideally matched to the operation of high-gain x-ray freeelectron lasers $[2,3]$. The transverse normalized emittance of an electron beam is properly matched to an x-ray beam if

$$
\gamma \varepsilon_{x} \quad \text { and } \quad \gamma \varepsilon_{y} \leq \frac{1}{4 \pi} \gamma \lambda_{r} .
$$

Here $\gamma$ is the electron energy in units of electron rest energy, and $\lambda_{r}$ is the free-electron laser (FEL) wavelength. For a $1-\AA$ FEL driven by a $15-\mathrm{GeV}$ electron beam, the normalized emittance of the matched electron beam is about $0.2 \mu \mathrm{m}$. However, the normalized transverse emittance from a state-of-the-art laser-driven rf photocathode gun is about an order of magnitude larger at about $1-n C$ charge:

$$
\gamma \varepsilon_{x, y}^{G} \approx 1 \mu \mathrm{m} .
$$

At lower charge the transverse emittance is limited by thermal emittance associated with the photoemission process. On the other hand, the uncorrelated rms energy spread from the $\mathrm{rf}$ gun $\sigma_{E}^{G}$ is quite small. The average value measured experimentally for a 4-nC charge is about $4 \mathrm{keV}$ [4]. For a much lower charge beam considered in this paper, we will assume

$$
\sigma_{E}^{G} \approx 1.5 \mathrm{keV},
$$

or, in terms of the relative energy spread immediately after the gun at an energy $E_{G} \approx 6 \mathrm{MeV}$ :

$$
\sigma_{\delta}^{G} \equiv \sigma_{E}^{G} / E_{G} \approx 2.5 \times 10^{-4} .
$$

Here $\sigma_{\delta}^{G}$ is the rms relative energy spread that, when compressed by up to a factor of 100 and accelerated to $15 \mathrm{GeV}$, is no more than $1 \times 10^{-5}$, or one order of magnitude smaller than necessary for avoiding gain reduction in an x-ray FEL. With the typical rms bunch length of $\sigma_{z} \approx$ $20 \mu \mathrm{m}$ (about $4 \mathrm{kA}$ of peak current at a $1-\mathrm{nC}$ bunch charge) and taking an increased energy spread of $\sigma_{\delta} \approx 1 \times 10^{-4}$ at $15 \mathrm{GeV}$, the normalized longitudinal emittance is about $\gamma \sigma_{\delta} \sigma_{z} \approx 60 \mu \mathrm{m}$. In fact, this order-of-magnitude energy spread increase is necessary to suppress the microbunching instability associated with the bunch compression process (see Ref. [5] and references therein). Thus we should ask whether it is possible to trade the excessively small energy spread with the transverse emittance, so that the transverse normalized emittance can be reduced to $0.1 \mu \mathrm{m}$ or smaller. We find that the answer is affirmative by a beam manipulation involving the flat-beam technique and emittance exchange.

In Sec. II, we first describe how a beam of normalized transverse and longitudinal emittance of, respectively, $1 \mu \mathrm{m}$ and $0.1 \mu \mathrm{m}$ with a peak current of $100 \mathrm{~A}$ at the gun cathode can be manipulated to produce a beam of normalized transverse and longitudinal emittance of, respectively, $0.1 \mu \mathrm{m}$ and $10 \mu \mathrm{m}$ with a peak current of $1 \mathrm{kA}$. We then show that the manipulated beam improves the performance of $\mathrm{x}$-ray FELs significantly. It is shown that 
the space-charge effect does not degrade the cathode emittance due to the fact that the beam at the cathode is a pancake shape with a large aspect ratio. We also show that the parameters for the flat-beam technique are reasonable. In Sec. III, we describe an optical arrangement giving rise to the exact emittance exchange necessary for our manipulation. The discussion up to this point is based on analytical arguments on linear transport elements. To estimate effects associated with a practical system, such as the nonlinearities, we have performed a simulation study of the beam manipulation in Sec. IV. Since the beams with a large aspect ratio considered in Sec. II are difficult to handle, we started from a cathode beam with smaller transverse emittance and less charge than that considered in Sec. II in this proof-of-principle simulation study. The simulated performance of the injector, consisting of a photocathode gun and a flat-beam transformer, is found to be close to the ideal case even after taking into account the space-charge effect. The simulation of the exchanger reveals that the higher-order effects are not negligible. Fortunately, however, it turns out that those effects can be made to cancel each other by adjusting the transverse-longitudinal correlation present in the injector output. Also, the emittance degradation due to the coherent synchrotron radiation turns out to be not serious. Section V concludes the paper, noting that the emittance exchanger has some interesting consequences on beam jitter, the beam produced in Sec. IV is not round but has a good FEL performance, and the beam manipulation technique proposed here has other applications, such as obviating the electron damping ring in a linear collider.

\section{THE PHASE-SPACE MANIPULATION SCHEME}

\section{A. The scheme}

We begin with the 4D transverse emittance from the gun, given by Eq. (2). By using the flat-beam technique, we change the partition of the emittances so that

$$
\left(\gamma \varepsilon_{x}, \gamma \varepsilon_{y}\right) \rightarrow(10,0.1) \mu \mathrm{m} .
$$

The beam current at the gun is taken to be the same as the linac coherent light source (LCLS) value

$$
I_{G}=100 \mathrm{~A} \text {. }
$$

The rms length of the bunch at the gun is chosen to be

$$
\sigma_{z}^{G}=34 \mu \mathrm{m}
$$

so that the longitudinal emittance in view of Eq. (3) becomes

$$
\gamma \varepsilon_{z}^{G}=\sigma_{z}^{G} \frac{\sigma_{E}^{G}}{m c^{2}} \approx 0.1 \mu \mathrm{m} .
$$

This represents a bunch charge of about $40 \mathrm{pC}$. We then exchange the $x$ and $z$ emittances to obtain

$$
\left(\gamma \varepsilon_{x}, \gamma \varepsilon_{y}, \gamma \varepsilon_{z}\right) \rightarrow(0.1,0.1,10) \mu \mathrm{m} .
$$

At some convenient point before the undulator, the beam is adjusted in the longitudinal phase space (bunch compression) so that the energy spread is increased to the maximum allowed by the condition that FEL gain is not degraded:

$$
\sigma_{\delta} \approx 1 \times 10^{-4},
$$

which is smaller than the dimensionless FEL parameter $\rho$ (typically around $5 \times 10^{-4}$ for an x-ray FEL). For a 15 $\mathrm{GeV}$ beam, the rms energy spread is then $1.5 \mathrm{MeV}$, and the rms bunch length after the adjustment is

$$
\sigma_{z} \approx 3.4 \mu \mathrm{m} \text {. }
$$

This is 10 times shorter than the bunch length at the gun. The beam current at the FEL will therefore increase by a factor of 10 :

$$
I_{f} \approx 1.0 \mathrm{kA} \text {. }
$$

\section{B. Application for x-ray FELs}

To show the usefulness of the phase-space manipulation, we consider a "Green field" FEL [1] operated at $\lambda_{r}=$ $0.4 \AA$. A permanent magnet undulator with a period of $3 \mathrm{~cm}$ is used. We also assume that the beta function in the undulator is optimized to produce the shortest gain length. Two possible beam configurations are considered. The electron beam in case (a) is similar to the LCLS, i.e., $\gamma \varepsilon_{x}=1 \mu \mathrm{m}$ and a peak current $I_{f}=3.5 \mathrm{kA}$ after bunch compression, while the electron beam in case (b) is the manipulated one as discussed above, i.e., $\gamma \varepsilon_{x}=0.1 \mu \mathrm{m}$ and a peak current $I_{f}=1.0 \mathrm{kA}$. Both beams have the same uncorrelated relative energy spread at $1 \times 10^{-4}$. Figure 1 shows the power gain length computed from Xie's fitting

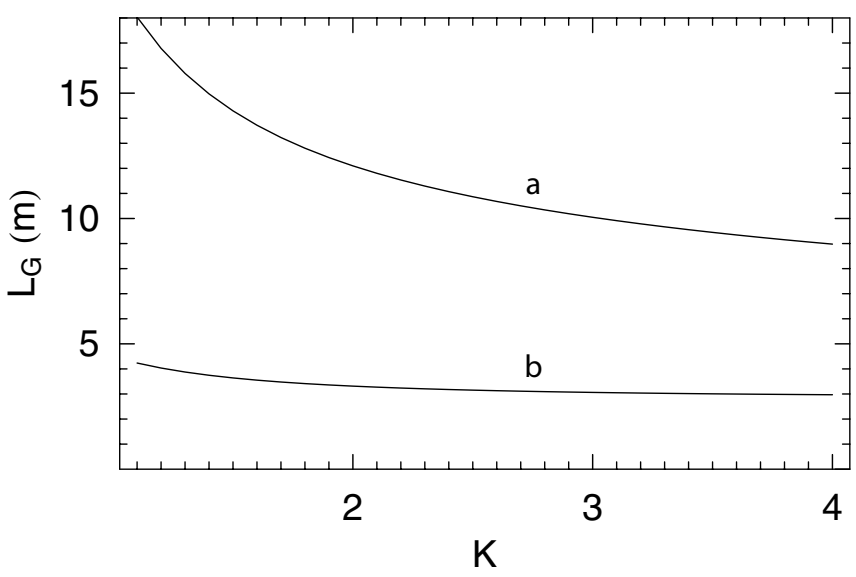

FIG. 1. Power gain length $L_{G}$ of an X-ray FEL at $0.4 \AA$ versus the undulator parameter $K$ for (a) a beam with a normalized transverse emittance of $1 \mu \mathrm{m}$ and a peak current of $3.5 \mathrm{kA}$, and (b) a beam with a normalized transverse emittance of $0.1 \mu \mathrm{m}$ and a peak current of $1.0 \mathrm{kA}$. The electron beam energy in both cases is adjusted according to the FEL resonant condition, with the relative rms energy spread at $1 \times 10^{-4}$. 
formula [6] versus the undulator parameter $K$ for both cases. The gain length for the case (b) is smaller by a factor of 2 or more. The length of the undulator will be reduced by a similar factor, reducing the construction cost as well as the operational complexity.

Note also that the gain length for case (b) changes little as $K$ increases from 1.5 to 4 , while it decreases significantly for case (a). Therefore, the $K$ value may be chosen to be small, $K \approx 1.5$, for the latter case while it needs to be large, $K \approx 4$, for the former case in order to limit the length of the undulator. The smaller emittance therefore has additional advantages: the undulator requires less magnetic material, and the electron energy required for $0.4-\AA$ radiation is smaller.

\section{Space-charge effects on the gun emittances}

Let us now consider in more detail some of the processes in the above manipulation of the phase-space distribution. Is the small longitudinal emittance, Eq. (8), consistent with the space-charge force? The emittance arising from the space-charge effect in the rf photocathode gun can be written as [7]

$$
\gamma \varepsilon_{i}^{\mathrm{SC}}=\frac{\pi}{4} \frac{1}{\sin \phi_{0}} \frac{2 m c^{2}}{E_{0}} \frac{I_{G}}{I_{A}} \mu_{i}(A)
$$

Here $i=x, y$, or $z ; \phi_{0}$ is the emission phase; $E_{0}$ is the peak electric field; $I_{A} \approx 17 \mathrm{kA}$ is the Alfven current; and $\mu_{i}(A)$ is a form factor depending on the aspect ratio $A=\sigma_{x}^{G} / \sigma_{z}^{G}$. The approximate formulas of the form factor for a Gaussian beam are [7]

$$
\begin{gathered}
\mu_{x, y}(A)=\frac{1}{3 A+5}, \\
\mu_{z}(A)=\frac{1.1}{1+4.5 A+2.9 A^{2}} .
\end{gathered}
$$

If we use the spot sizes $\sigma_{x}^{G}=0.6 \mathrm{~mm}$ and $\sigma_{z}^{G}=34 \mu \mathrm{m}$, we obtain $A \approx 18$, a large aspect ratio making the form factor very small. Using the LCLS values for the peak gun field $E_{0}=120 \mathrm{MV} / \mathrm{m}, \quad I_{G}=100 \mathrm{~A}$, and assuming $\pi /\left(4 \sin \phi_{0}\right) \approx 1$, we obtain

$$
\begin{aligned}
& \gamma \varepsilon_{x, y}^{\mathrm{SC}} \approx 1.0 \mu \mathrm{m} \\
& \gamma \varepsilon_{z}^{\mathrm{SC}} \approx 0.1 \mu \mathrm{m} .
\end{aligned}
$$

Therefore the longitudinal emittance due to the spacecharge effect is indeed very small and consistent with Eq. (7). The transverse emittance is also small, consistent with Eq. (2), implying that the emittance compensation [8] may not be necessary for a beam with such large aspect ratio and low charge.

\section{The flat-beam technique}

The flat-beam technique is a method to produce two dissimilar emittances starting from a round beam $[9,10]$. It begins with the process in which an electron beam in thermal motion inside a cathode is born into a region with an axial magnetic field $B$, suddenly acquiring canonical angular momentum. The beam is then brought into a fieldfree region and the angular momentum is removed by a triplet of skew quadrupole magnets [11]. In this process the initially equal emittances, Eq. (2), are partitioned into unequal emittances $\left(\gamma \varepsilon_{+}, \gamma \varepsilon_{-}\right)$, preserving the product: $\gamma \varepsilon_{x} \times \gamma \varepsilon_{y}=\gamma \varepsilon_{+} \times \gamma \varepsilon_{-}$. An unequal partitioning of equal emittances is not possible in a symplectic process; in a symplectic process the emittances in different directions can either remain the same or be exchanged among themselves [12]. The flat-beam technique avoids this limitation because the process of beam birth in a magnetic field is nonsymplectic. The technique was theoretically analyzed in Refs. [13,14].

The emittance ratio after the flat-beam technique is given by

$$
\frac{\varepsilon_{+}}{\varepsilon_{-}} \approx\left(\frac{e B\left(\sigma_{x}^{G}\right)^{2}}{m c \gamma \varepsilon_{x}^{G}}\right)^{2} \gg 1
$$

where $e$ is the electron charge, $m$ is the electron mass, and $c$ is the speed of light. The ratio is 100 in the present case and the required magnetic field is modest: $B=470 \mathrm{G}$. Experimentally, the flat-beam technique was demonstrated at the Fermilab/NICADD [15] Photo Injector Laboratory [16-19]. The best partition of emittances recently achieved is $\left(\gamma \varepsilon_{+}, \gamma \varepsilon_{-}\right) \approx(40,0.4) \mu \mathrm{m}[20]$. Note that the emittance ratio is 100 , although the product is an order of magnitude larger than what is needed for the scheme in this paper.

\section{OPTICS FOR EMITTANCE EXCHANGE}

A study of emittance exchange has been performed [21] with the beam transport system, which produces the transverse-to-longitudinal emittance exchange constructed from a four-dipole chicane and a dipole-mode cavity. The first half of the chicane (a dogleg bend) consists of a pair of bending magnets of opposite polarities separated by a free space of length $L$ with the $x-z 4 \times 4$ transfer matrix (ignoring the vertical, or non-bend-plane coordinate):

$$
\mathbf{M}_{D}(\eta, \xi, L)=\left[\begin{array}{llll}
1 & L & 0 & \eta \\
0 & 1 & 0 & 0 \\
0 & \eta & 1 & \xi \\
0 & 0 & 0 & 1
\end{array}\right] .
$$

Here $\eta$ and $\xi$ are, respectively, the dispersion and the momentum compaction generated in the dogleg. The transfer matrix for a thin-lens dipole-mode cavity is of the form [21] 


$$
\mathbf{M}_{C}(k)=\left[\begin{array}{cccc}
1 & 0 & 0 & 0 \\
0 & 1 & k & 0 \\
0 & 0 & 1 & 0 \\
k & 0 & 0 & 1
\end{array}\right],
$$

where $k$ is related to the cavity voltage $V_{0}$, cavity dimension $a$, and beam energy in the cavity $E_{0}$ by

$$
k \equiv \frac{V_{0}}{a E_{0}} .
$$

The emittance exchange scheme studied in Ref. [21] consists of a dipole-mode cavity flanked by two doglegs of opposite sign (i.e., the cavity is in the middle of a chicane) with the transfer matrix:

$$
\mathbf{M}_{\mathrm{EX}}=\mathbf{M}_{D}(-\eta, \xi, L) \mathbf{M}_{C}(k) \mathbf{M}_{D}(\eta, \xi, L) .
$$

It was shown that Eq. (22) produces an approximate emittance exchange if $1-(k \eta)^{2}=0$. The exchange in this system is not perfect, due to a residual transverse-tolongitudinal coupling term, although this was not a significant limitation with the 5-to-1 emittance exchange ratio described in Ref. [21]. However, as the larger initial emittance is increased $\left(\gamma \varepsilon_{x_{o}}=10 \mu \mathrm{m}\right)$ and the smaller initial emittance is decreased $\left(\gamma \varepsilon_{z_{o}}=0.1 \mu \mathrm{m}\right)$, the limitation due to imperfect exchange becomes significant. The residual coupling term can be reduced to arbitrarily small levels by decreasing the dispersion in the chicane (i.e., weaker chicane bends and stronger rf kick). However, a secondorder dispersion aberration arises after the cavity, which is inversely proportional to the dispersion and therefore limits this coupling reduction.

Fortunately, an exact exchange optics was found by one of us (K.-J. K.) [22] that is similar to that described above, but with the second dogleg identical to the first one as shown in Fig. 2. The corresponding transfer matrix is

$$
\mathbf{M}_{\mathrm{EX}}=\mathbf{M}_{D}(\eta, \xi, L) \mathbf{M}_{C}(k) \mathbf{M}_{D}(\eta, \xi, L) .
$$

If the dispersion $\eta$ and the kick strength $k$ are chosen so that

$$
1+k \eta=0
$$

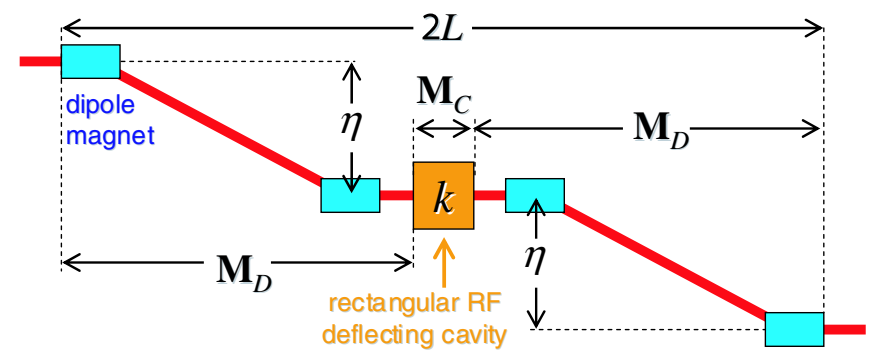

FIG. 2. (Color) Schematic diagram for exact longitudinal-totransverse emittance exchange, consisting of two identical doglegs $\left(\mathbf{M}_{D}\right)$ and a transverse cavity $\left(\mathbf{M}_{C}\right)$. The matrix for $\mathbf{M}_{D}$ and $\mathbf{M}_{C}$ are given by Eqs. (19) and (20), respectively. it is then easy to show that the $4 \times 4 x-z$ transfer matrix becomes

$$
\mathbf{M}_{\mathrm{EX}}=\left[\begin{array}{ll}
\mathbf{0} & \mathbf{B} \\
\mathbf{C} & \mathbf{0}
\end{array}\right]
$$

where

$$
\mathbf{B}=\left[\begin{array}{cc}
k L & \eta+k L \xi \\
k & k \xi
\end{array}\right], \quad \mathbf{C}=\left[\begin{array}{cc}
k \xi & \eta+k L \xi \\
k & k L
\end{array}\right] .
$$

The transfer matrix, Eq. (25), is in the form of an exact emittance exchange.

We introduce the $4 \times 4$ beam matrix $\boldsymbol{\Sigma}$, whose elements are the second central moments of phase-space variables:

$$
\Sigma_{i j}=\left\langle X_{i} X_{j}\right\rangle
$$

Here $\langle\cdots\rangle$ represents taking the average over the ensemble of particles and $\left(X_{1}, X_{2}, X_{3}, X_{4}\right) \equiv\left(x, x^{\prime}, z, \delta\right)$. The $4 \times 4$ beam matrix before the exchange is

$$
\boldsymbol{\Sigma}_{b}=\left[\begin{array}{cc}
\boldsymbol{\Sigma}_{x} & 0 \\
0 & \boldsymbol{\Sigma}_{z}
\end{array}\right]
$$

where the $2 \times 2$ submatrices are

$$
\boldsymbol{\Sigma}_{x}=\varepsilon_{x}\left[\begin{array}{cc}
\beta_{x} & 0 \\
0 & 1 / \beta_{x}
\end{array}\right], \quad \boldsymbol{\Sigma}_{z}=\varepsilon_{z}\left[\begin{array}{cc}
\beta_{z} & 0 \\
0 & 1 / \beta_{z}
\end{array}\right],
$$

and $\beta_{x}$ and $\beta_{z}$ are the beta functions in the $x$ - and $z$-directions, respectively. After the exchange the beam matrix becomes

$$
\boldsymbol{\Sigma}_{a}=\mathbf{M}_{\mathrm{EX}} \boldsymbol{\Sigma}_{b} \mathbf{M}_{\mathrm{EX}}{ }^{T}=\left[\begin{array}{cc}
\mathbf{B} \boldsymbol{\Sigma}_{x} \mathbf{B}^{T} & \mathbf{0} \\
\mathbf{0} & \mathbf{C} \boldsymbol{\Sigma}_{z} \mathbf{C}^{T}
\end{array}\right] .
$$

Here the superscript $T$ denotes the transpose operation.

\section{SIMULATION STUDY}

A numerical optimization of a photoinjector capable of providing the needed parameters has been performed. This numerical study is for a proof-of-principle demonstration that effects not included in the previous sections do not seriously degrade the performance of the phase-space manipulation scheme. Thus the specific parameters in this section are somewhat different than described above. In particular, we have chosen here to start from an electron beam with smaller transverse emittance and less longitudinal current to keep the transverse-longitudinal aspect ratio to a level that can be handled easily.

\section{A. The injector}

The electron source is a 1.5-cell radio-frequency (rf) gun operating at $2.6 \mathrm{GHz}$ producing a $7.5-\mathrm{MeV}$ electron bunch. The gun is surrounded by two magnetic solenoids. One provides a nonzero field on the photocathode surface, as needed for producing an angular-momentum-dominated 
beam, while the other solenoid allows control of the beam envelope. A downstream linear accelerator (linac) composed of eight TESLA-type superconducting cavities operating at $1.3 \mathrm{GHz}$ increases the beam energy to approximately $215 \mathrm{MeV}$. The linac is followed by a round-to-flatbeam converter composed of three skew quadrupole magnets that transforms the incoming angular-momentumdominated beam into a flat, uncoupled beam.

The photoinjector was modeled with the particle-in-cell tracking programs ASTRA [23] and IMPACT-T [24]. ASTRA was used to model the cylindrically symmetric portion of the beam line. The axial field profiles were used to model the cavities and solenoid, and the radial dependence of the longitudinal accelerating fields was taken into account up to second order in radius. A low longitudinal emittance is achievable provided nonlinearities in the longitudinal phase space are kept insignificant. To this end we assumed the photocathode was illuminated by a 3D oblate ellipsoid laser pulse [25]. Because of the quadratic dependence of the accelerating field on radial position, a small longitudinal emittance calls for a small initial beam radius on the photocathode. Such a requirement results in a small transverse emittance whose main contribution comes from the photoemission process. The thermal emittance was taken to be $0.6 \mu \mathrm{m}$ per $\mathrm{mm}$ rms transverse size on the photocathode, a value consistent with an excess kinetic energy of $0.26 \mathrm{eV}$ for copper cathode. Because of the short response time of the metallic-type photocathode, the photocathode drive-laser pulse duration can in principle be very short (less than $50 \mathrm{fs}$ ). The longitudinal emittance was mini-

TABLE I. Operating parameters and achieved beam parameters at the photoinjector end.

\begin{tabular}{lcc}
\hline \hline Operating parameters & Value & Units \\
\hline Bunch charge & 20 & $\mathrm{pC}$ \\
Laser rms spot size & 300 & $\mu \mathrm{m}$ \\
Laser rms pulse duration & 80 & $\mathrm{fs}$ \\
Peak $E$-field in rf-gun & 138 & $\mathrm{MV} / \mathrm{m}$ \\
Launch phase & 45 & $\mathrm{deg}$ \\
Peak -field in TESLA cavities & 36 & $\mathrm{MV} / \mathrm{m}$ \\
B-field on photocathode & 0.191 & $\mathrm{~T}$ \\
Cavity off-crest phase & 4 & $\mathrm{deg}$ \\
\hline Beam parameters & Value & $\mathrm{Units}$ \\
\hline Before flat-beam transformation & & \\
Transverse (coupled) projected $x$ and & 4.96 & $\mu \mathrm{m}$ \\
$\quad y$ emittances & & \\
Intrinsic transverse $x$ and $y$ emittances & 0.23 & $\mu \mathrm{m}$ \\
Longitudinal emittance & 0.071 & $\mu \mathrm{m}$ \\
Kinetic energy & 215.4 & $\mathrm{MeV}$ \\
After round-to-flat-beam transformation & & \\
Emittance $\gamma \varepsilon_{x}$ & 9.9 & $\mu \mathrm{m}$ \\
Emittance $\gamma \varepsilon_{y}$ & 0.0054 & $\mu \mathrm{m}$ \\
Longitudinal emittance & 0.080 & $\mu \mathrm{m}$ \\
$\gamma\left(\varepsilon_{x} \varepsilon_{y}\right)^{1 / 2}$ & 0.23 & $\mu \mathrm{m}$ \\
\hline \hline
\end{tabular}

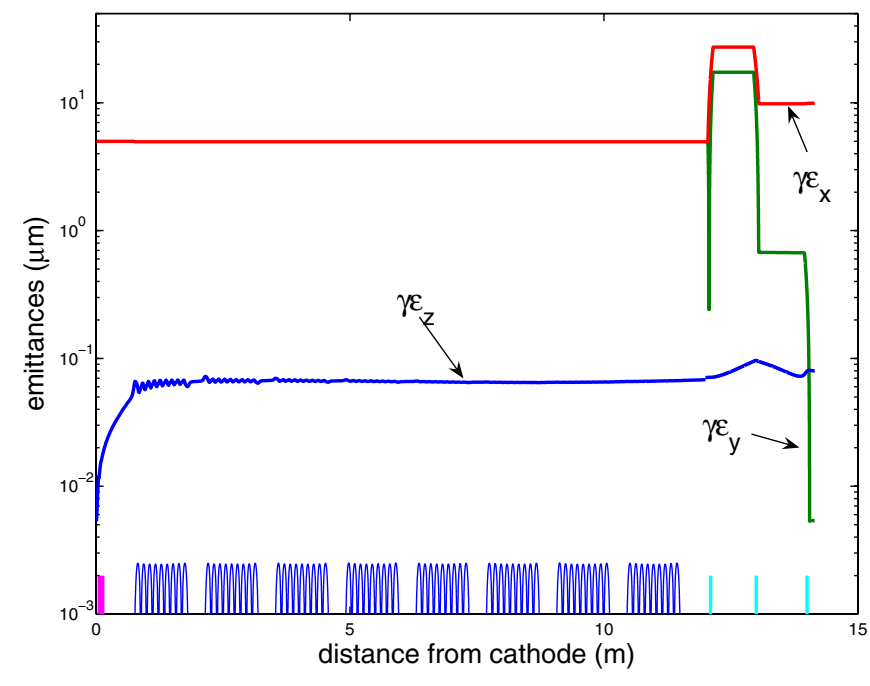

FIG. 3. (Color) Evolution of the transverse horizontal (red), vertical (green), and longitudinal (blue) emittances along the photoinjector beam line. The purple and cyan boxes at the bottom indicate the locations of the rf gun and the skew quadrupoles, respectively, while the blue lines indicate the accelerating cavity contour.

mized with the constraint of generating a short electron bunch (50 $\mu \mathrm{m}$ or less) downstream of the accelerating module. The minimization was performed with the generic optimizer SDDSOPTIMIZE [26]. The optimized phase space was then passed to the program IMPACT-T to simulate the round-to-flat-beam transformation, taking into account space-charge forces. The operating parameters and achieved beam parameters are summarized in Table I. The evolution of the beam emittances along the photoinjector beam line is shown in Fig. 3.

\section{B. The emittance exchanger}

The emittance exchange was simulated using ELEGANT [27], including second-order optical aberrations, by tracking $2 \times 10^{5}$ macroparticles - taken from the output of the injector simulation - through the system shown in Fig. 2 and using the parameters listed in Table II. Transverse and longitudinal phase-space plots are shown in Fig. 4, both before and after the emittance exchange system, without yet including the effects of coherent synchrotron radiation (CSR) in the dipole magnets. The nearly perfect emittance exchange is achieved only after minimizing both the thicklens and second-order effects with a unique incoming energy chirp ( $\delta-z$ slope $)$ of the electron beam given by

$$
h=-\frac{1}{\xi} .
$$

Limitations introduced by a finite rf cavity length have already been described in Ref. [21], where a specific initial energy chirp is used to compensate this effect. In addition, a second-order dispersion error also arises, which was 
TABLE II. Emittance exchange simulation parameters.

\begin{tabular}{lccc}
\hline \hline \multicolumn{1}{c}{ Parameter } & Symbol & Value & Unit \\
\hline Electron energy & $E$ & 215 & $\mathrm{MeV}$ \\
Dipole magnet length & $L_{B}$ & 20 & $\mathrm{~cm}$ \\
Drift length between dipole magnets & $L$ & 1 & $\mathrm{~m}$ \\
Bend angle per dipole magnet & $\theta$ & 20 & $\mathrm{deg}$ \\
Length of rf cavity & $L_{c}$ & 30 & $\mathrm{~cm}$ \\
Initial horizontal norm. emittance & $\gamma \varepsilon_{x 0}$ & 9.9 & $\mu \mathrm{m}$ \\
Initial longitudinal norm. emittance & $\gamma \varepsilon_{z 0}$ & 0.080 & $\mu \mathrm{m}$ \\
Initial rms bunch length & $\sigma_{z 0}$ & 51 & $\mu \mathrm{m}$ \\
Initial rms slice energy spread & $\sigma_{E} G$ & 0.9 & $\mathrm{keV}$ \\
Initial energy chirp ( $\delta$ - $z$ slope) & $h$ & 6.86 & $\mathrm{~m}$ \\
Initial horizontal beta function & $\beta_{x 0}$ & 100 & $\mathrm{~m}$ \\
Initial horizontal alpha function & $\alpha_{x 0}$ & 0 & \\
Final horizontal norm. emittance & $\gamma \varepsilon_{x f}$ & 0.16 & $\mu \mathrm{m}$ \\
(including CSR estimation, see text) & & & \\
Final longitudinal norm. emittance & $\gamma \varepsilon_{z f}$ & 11 & $\mu \mathrm{m}$ \\
\hline \hline
\end{tabular}

briefly described in Ref. [21]. The additional energy spread introduced by the rf cavity must pass through the two remaining bends of the exchanger system. Although the linear dispersion is fully included in the linear exchanger theory in Eq. (19), the second-order dispersion of the final two bends is not included and can introduce another limitation. This effect can also be compensated by the same initial energy chirp introduced to compensate the thicklens cavity. The second-order dispersion aberration is purely spatial, $\Delta x$, immediately after the last bend, and scales as $\Delta x \sim \delta^{2}$, where $\delta$ is the additional energy deviation caused by an off-axis particle in the cavity. As in any purely spatial aberration, the associated emittance growth can always be minimized by choosing a large beta function at that point, so that the spatial aberration becomes small in comparison to the beam size. The initial energy chirp [given by Eq. (31)] that compensates the thick-lens cavity effect also maximizes the horizontal beta function after the last bend, minimizing the second-order dispersion aberration to an insignificant level.

To minimize the effects of CSR in the double doglegs, we have chosen a large initial bend-plane $(x)$ beta function (100 $\mathrm{m}$ as shown in Table II) at the beginning of the exchanger system by rematching the horizontal beam size from the injector simulation. Such a large beta function, together with a large horizontal emittance $(9.9 \mu \mathrm{m})$, increases the effective rms bunch length in the system. For
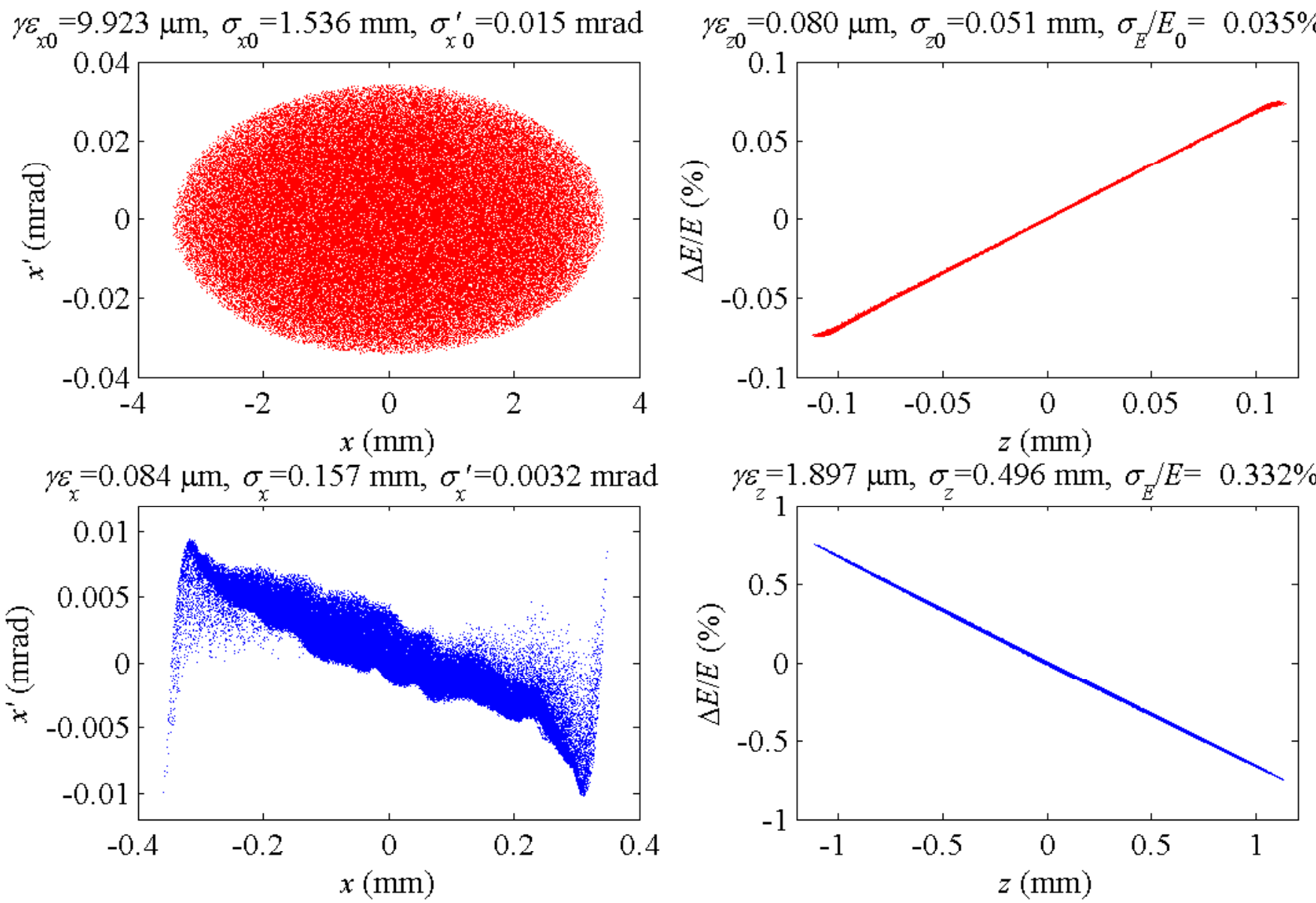

FIG. 4. (Color) Transverse phase space (left two plots) and longitudinal phase space (right two plots) before (top row) and after (bottom row) the emittance exchange as simulated with ELEGANT, using particles from the injector simulation, but not including CSR. 
example, the effective rms bunch length in the first dipole is given by

$$
\sigma_{z}(s)=\sqrt{\sigma_{z 0}^{2}+\frac{s^{2}}{\rho^{2}} \sigma_{x}^{2}}=\sqrt{\sigma_{z 0}^{2}+\frac{s^{2}}{\rho^{2}} \varepsilon_{x 0} \beta_{x 0}},
$$

where $\sigma_{z 0}$ is the initial bunch length, and $s$ is the distance traveled along the dipole magnet with bend radius $\rho$. Figure 5 shows both the bunch length and the energy spread evolution along the exchanger beam line. The effective bunch length remains much longer than the initial bunch length, except for a very short section between the end of the second dipole and the beginning of the third dipole, where the rms bunch length reaches $\sim 7 \mu \mathrm{m}$ due to the special choice of the initial energy chirp (to suppress thick-lens and second-order effects). As a result of the larger average bunch length in the system, and with only $20 \mathrm{pC}$ of charge, the CSR-induced bend-plane emittance growth computed using the 1D CSR algorithm of ELEGANT is found to be modest, about a factor of 2.5. In addition, the CSR effect calculated by 1D formulas may give an overestimate of the emittance growth when the bunch is extremely short because the transverse coherence length of the radiation may be much smaller than the horizontal beam size [i.e., when $\left(\rho \sigma_{z}^{2}\right)^{1 / 3} \ll \sigma_{x}$ at the end of the second dipole and the beginning of the third dipole, see Fig. 5] [28]. Since this 3D-suppression effect is not included in the 1D CSR model of ELEGANT, we use a factor of 2 instead of 2.5 to estimate the final horizontal emittance as shown in Table II.

Although the large beta function helps suppress the CSR wakefield, the disadvantage is that the final $\mathrm{rms}$ bunch length is increased to about $500 \mu \mathrm{m}$ after the emittance exchange (see Figs. 4 and 5). Thus, the final peak current in this numerical example is only $3.4 \mathrm{~A}$. The bunch needs to be further compressed by a factor of 150 to 300 in order to be useful for a short-wavelength FEL. This compression

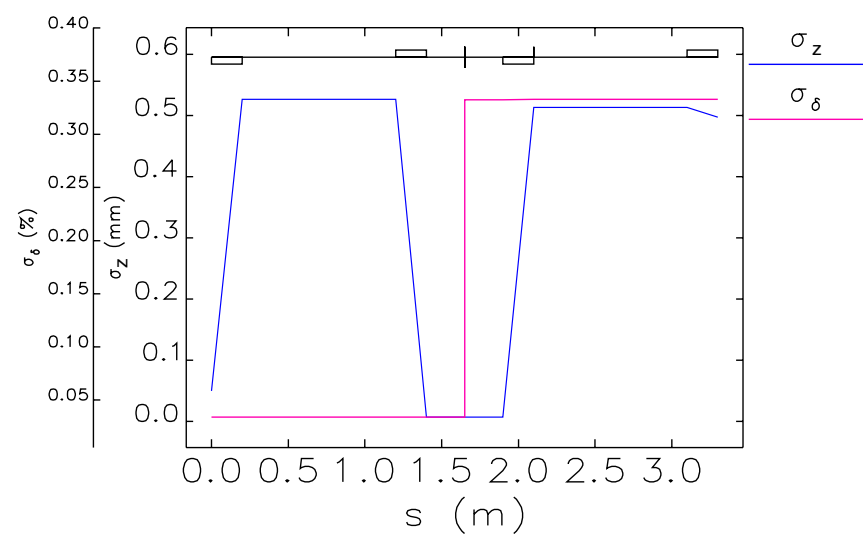

FIG. 5. (Color) The rms bunch length and energy spread in the emittance exchange system where the effective bunch length is quite long during most of the transport, due to the large initial bend-plane beta function and emittance. may be accomplished in multiple stages at higher beam energies and with weaker bends, in order to minimize any additional CSR effects.

\section{FURTHER REMARKS AND CONCLUSIONS}

One interesting aspect of this system is that the exchanger is a timing-jitter and an energy-jitter "absorber." If the bunch arrival time changes at the entrance to the exchanger, this timing error completely disappears at the exit of the exchanger. In fact, it does not actually disappear, but simply transforms into an offset in the transverse angle-just as the emittance values are exchanged, so are the centroid variations. Although this may be an asset from the point of view of synchronization or energy stability, it may be a drawback from the point of view of the transverse stability. Even with a very small level of $1 \times$ $10^{-5}$ relative energy jitter at the entrance to the exchanger $(2 \mathrm{keV})$, the transverse amplitude jitter will become 2 times the rms transverse beam size. This may be a serious problem for trajectory and x-ray pointing stability in the FEL.

This jitter transfer issue is a fundamental one and cannot be arbitrarily minimized. The complete longitudinal-totransverse coordinate exchange, and its reverse, means that a one-sigma centroid variation in the longitudinal coordinates (time and energy) must become a one-sigma centroid variation in the transverse coordinates (position and angle). The only mitigation is to reduce the source of the jitter, or to increase the value of one-sigma of the least stable longitudinal coordinate, while preserving the longitudinal emittance. For example, reducing the initial bunch length from 50 to $25 \mu \mathrm{m}$ and increasing the initial slice energy spread from 1 to $2 \mathrm{keV}$, will make the system more tolerant of energy jitter by a factor of 2 . However, it will be less tolerant of timing jitter by the same factor. This is similar to what occurs in the final focusing doublet of a linear collider, where a very large beta function in the doublet produces a very small angular divergence. The small divergence, analogous to the small slice energy spread, makes the beam very sensitive to tiny kick angles due to quadrupole vibrations, setting very demanding tolerances on doublet mechanical stability.

In any case, these jitter issues will become always more challenging in any machine which requires smaller emittance levels, requiring technical advances in the stability of all systems which go hand-in-hand with advances in emittance.

The FEL performance typically optimizes for a round beam (with equal transverse emittances) for the same emittance product. In this proof-of-principle simulation study, our beam remains flat even after the emittance exchange due to the extremely small vertical emittance $\left(\gamma \varepsilon_{y}=0.0054 \mu \mathrm{m}\right)$. Although we believe further numerical optimization can yield a final round beam solution, we have not carried out these studies in this paper. Nevertheless, such a flat beam with a small horizontal 
emittance $\left(\gamma \varepsilon_{x}=0.16 \mu \mathrm{m}\right)$ and an even smaller vertical emittance is already useful for driving a Green field FEL at $\lambda_{r}=0.4 \AA$ as discussed earlier. The flat-beam FEL performance was discussed by Xie in Ref. [29]. In our case, for a final compressed peak current of $1 \mathrm{kA}$ and an average $x / y$ beta function of $20 \mathrm{~m}$ in the undulator, the flat beam yields a power gain length of $4 \mathrm{~m}$ at $K=1.34$ (at electron energy $13.6 \mathrm{GeV}$ ), which is still a factor of 2 smaller than a round beam with both transverse normalized emittance at $0.16 \mu \mathrm{m}$.

Finally, the technique presented in the present paper may also find application in a linear $e^{+} / e^{-}$collider, which requires flat beams at the collision point. The original flat-beam gun proposal [10] does not seem to meet the International Linear Collider design goal of $\left(\gamma \varepsilon_{x}, \gamma \varepsilon_{y}\right) \rightarrow$ $(8,0.02) \mu \mathrm{m}$ at $3.2 \mathrm{nC}$. It is conceivable to exchange the larger (than $8 \mu \mathrm{m}$ ) horizontal emittance with a smaller longitudinal emittance through flat-beam generation and emittance exchange described in this paper to reach this design goal at such a high charge. Further investigations including the issues of generating spin-polarized electrons will be the subject of future work.

\section{ACKNOWLEDGMENTS}

Work at Argonne was supported by the U.S. Department of Energy, Office of Science, Office of Basic Energy Sciences, under Contract No. W-31-109-ENG-38; at SLAC by the U.S. Department of Energy, Contract No. DE-AC02-76SF00515; and at NIU and Fermilab by the Northern Illinois Center for Accelerator and Detector Development (NICADD) and the Universities Research Association Inc. under DOE Contract No. DE-AC0276CH00300, respectively.

[1] K.-J. Kim, J. Galayda, and J. Murphy, presentation at BESAC Subcommittee on BES 20-Year Facility Road Map, Washington, D.C., 2003.

[2] Linac Coherent Light Source Conceptual Design Report, SLAC-R-593, 2002.

[3] TESLA XFEL Technical Design Report Supplement, TESLA FEL 2002-09, 2002.

[4] M. Hüning and H. Schlarb, Proceedings of the 2003 Particle Accelerator Conference, Portland, OR (2003), pp. 2074-2076.

[5] Z. Huang et al., Phys. Rev. ST Accel. Beams 7, 074401 (2004).
[6] M. Xie, Proceedings of the 1995 Particle Accelerator Conference, Dallas, TX (1996), pp. 183-185.

[7] K.-J. Kim, Nucl. Instrum. Methods Phys. Res., Sect. A 275, 201 (1989).

[8] B. E. Carlsten, Nucl. Instrum. Methods Phys. Res., Sect. A 285, 313 (1989).

[9] Ya Derbenev, University of Michigan Report No. UM-HE98-04, 1998.

[10] R. Brinkmann, Ya. Derbenev, and K. Floettmann, Phys. Rev. ST Accel. Beams 4, 053501 (2001).

[11] A. Burov and V. Danilov, Fermilab-TM-2043, 1998.

[12] E. Courant, in Perspectives in Modern Physics, Essays in Honor of Hans A. Bethe, edited by R.E. Marshak (Interscience Publishers, New York, 1966), pp. 257-260.

[13] A. Burov, S. Nagaitsev, and Ya Derbenev, Phys. Rev. E 66, 016503 (2002).

[14] K.-J. Kim, Phys. Rev. ST Accel. Beams 6, 104002 (2003).

[15] NICADD is an acronym for Northern Illinois Center for Accelerator and Detector Development.

[16] D. Edwards et al., Proceedings of the XX International Linac Conference, Monterey, CA (2000), pp. 122-124.

[17] D. Edwards et al., Proceedings of the 2001 Particle Accelerator Conference, Chicago, IL (IEEE, Piscataway, NJ, 2001), pp. 73-75.

[18] E. Thrane et al., Proceedings of the XXI International Linac Conference, Gyeungju, Korea (2002), pp. 308-310.

[19] Y.-E Sun et al., Phys. Rev. ST Accel. Beams 7, 123501 (2004).

[20] Ph. Piot, Y.-E Sun, and K.-J. Kim, Phys. Rev. ST Accel. Beams 9, 031001 (2006).

[21] M. Cornacchia and P. Emma, Phys. Rev. ST Accel. Beams 5, 084001 (2002).

[22] K.-J. Kim and A. Sessler, Proceedings of International Workshop on Beam Cooling and Related Topics, Galena, IL, USA, AIP Conf. Proc. No. 821 (AIP, New York, 2006), pp. $115-138$.

[23] ASTRA user manual available at http://www.desy.de/ mpyflo/Astra_dokumentation/

[24] J. Qiang et al., Proceedings of the 2005 Particle Accelerator Conference, Knoxville, TN (2005), pp. 3316-3318.

[25] O. J. Luiten et al., Phys. Rev. Lett. 93, 094802 (2004).

[26] H. Shang et al., Proceedings of the 2003 Particle Accelerator Conference, Portland, OR (2003), pp. 34703472.

[27] M. Borland, ANL Advanced Photon Source Report No. LS-287 (2000).

[28] G. Stupakov (private communication).

[29] M. Xie, Nucl. Instrum. Methods Phys. Res., Sect. A 507, 450 (2003). 